\title{
DEEP TRAPS SPECTRA IN UNDOPED GAN FILMS GROWN BY HYDRIDE VAPOR PHASE EPITAXY UNDER VARIOUS CONDITIONS
}

\author{
${ }^{1,2}$ Polyakov, A.Y., ${ }^{2,3}$ N.B. Smirnov, ${ }^{3}$ A.V. Govorkov, ${ }^{4}$ H. Cox,${ }^{4,5}$ A.S. Usikov, \\ ${ }^{4}$ H. Helava, ${ }^{4}$ Y.N. Makarov, ${ }^{2,6}$ E.B. Yakimov and ${ }^{1}$ I.H. Lee \\ ${ }^{1}$ School of Advanced Materials Engineering and Research Center of Advanced Materials Development, \\ Chonbuk National University, Jeonju, Korea \\ ${ }^{2}$ National University of Science and Technology MISiS, Moscow, Russia \\ ${ }^{3}$ Institute of Rare Metals, Moscow, Russia \\ ${ }^{4}$ Nitride Crystals, Inc., Deer Park, USA \\ ${ }^{5}$ Saint-Petersburg National Research University of Information Technologies, \\ Mechanics and Optics, Saint Petersburg, Russia \\ ${ }^{6}$ Institute of Microelectronics Technology and High Purity Materials Russian Academy of Science, \\ Chernogolovka, Russia
}

Received 2014-04-23; Revised 2014-07-24; Accepted 2014-09-12

\begin{abstract}
Decreasing the residual donors density and deep traps spectra densities in undoped GaN films grown by Hydride Vapor Phase Epitaxy (HVPE) is very important for promoting the use of such material in highvoltage/high-power rectifiers, radiation detectors. In this study we studied the effects of changing the growth temperature of undoped HVPE GaN films on these properties. The two groups of undoped $\mathrm{GaN}$ HVPE samples analyzed in this study were grown at growth temperature being either $850^{\circ} \mathrm{C}$ or $950^{\circ} \mathrm{C}$. Measurements by means of Capacitance-Voltage $(\mathrm{C}-\mathrm{V})$ profiling, deep levels transient spectroscopy, Micro Cathode Luminescence (MCL) spectroscopy and imaging and by Electron Beam Induced Current (EBIC) showed a much lower density of residual donors (by almost two orders of magnitude), of deep electron traps and hole traps (by about an order of magnitude) and considerably (about 1.5 times) longer diffusion length of charge carriers in the films grown at $850^{\circ} \mathrm{C}$ compared to samples prepared at $950^{\circ} \mathrm{C}$. The data obtained indicate that there is an optimal reduced growth temperature (close to $850^{\circ} \mathrm{C}$ ) resulting in lower concentration of shallow donors and deep traps while still preserving the high crystalline quality of the layer. This is of paramount importance for device applications of HVPE grown undoped GaN films.
\end{abstract}

Keywords: Deep Traps, Undoped GaN Films, HVPE

\section{INTRODUCTION}

Hydride vapor phase epitaxy is a well-established growth technique for fabrication of Group III-Nitrides thick films and bulk crystals with good crystalline quality (Liu et al., 2014; Wu et al., 2013; Sato et al., 2013; Paskova et al., 2010; Fujito et al., 2009;
Yoshida et al., 2008) The technique is quite versatile allowing to grow films and crystals of n-type (typically, by Si doping), p-type (by Mg doping) and semi-insulating (by deep levels $\mathrm{Fe}$ or $\mathrm{Zn}$ compensation) (Richter et al., 2013; Usikov et al., 2008; Lipski, 2010; Reshchikov et al., 2011). The growth rate can be varied from tens of nanometers per Corresponding Author: Usikov, A.S., School of Advanced Materials Engineering and Research Center of Advanced Materials Development, Chonbuk National University, Jeonju, Korea 
hour to hundreds of microns per hour. The dislocation density can be made as low as $10^{4}-10^{7} \mathrm{~cm}^{-2}$, depending on the layers thickness (Fujito et al., 2009; Sato et al., 2013). All this makes HVPE a very promising technique for fabrication of bulk $\mathrm{GaN}$ that can be used as substrates in nitrides epitaxy. HVPE GaN films and device structures can be also very useful for light emitting diodes, high-power rectifiers, transistors and radiation detectors (Dmitriev and Usikov, 2006; Reed et al., 2009; Kurin et al., 2013; Wang et al., 2011; Pearton et al., 2013) however, present day HVPE films still suffer from a rather high residual donor concentration (typically undoped GaN films are n-type with the uncompensated shallow donor density of $\sim 10^{17} \mathrm{~cm}^{-3}$ or even higher) and relatively high density of deep electron and, particularly, hole traps (Lee et al., 2012; Polyakov et al., 2011; Fujito et al., 2009; Yoshida et al., 2008), which seriously limits their applications in device structures. In what follows we analyze how the growth conditions (growth temperature especially) affect the electrical and optical properties and the deep traps spectra of HVPE layers.

\section{MATERIALS AND METHODS}

Undoped GaN films in this study were grown by HVPE on basal plane sapphire substrates. The growth process was performed in argon ambient with metallic $\mathrm{Ga}$ as a source material and hydrogen chloride and ammonia as active gases. The growth was performed at temperatures of $850-950^{\circ} \mathrm{C}$ that were somewhat lower than in a typical HVPE process of $1020-1050^{\circ} \mathrm{C}$ (Usikov et al., 2013; Sato et al., 2013; Fujito et al., 2009; Liu et al., 2014; Paskova et al., 2010) the growth rate was $0.2-1 \mu \mathrm{m} / \mathrm{min}$ and the total thickness of the layers was $10-20 \mu \mathrm{m}$. The growth was initiated at low growth rate and then continued with increased growth rate. The two groups of samples studied in this study mainly differed by the growth temperature. Group 1 samples were prepared at growth temperature of $\sim 950^{\circ} \mathrm{C}$ in a horizontal reactor, group 2 samples were grown at $\sim 850^{\circ} \mathrm{C}$ in a vertical reactor.

The grown films were characterized by capacitancevoltage $\mathrm{C}-\mathrm{V}$ profiling (HP4192A C-V meter for the $10 \mathrm{~Hz}-10 \mathrm{MHz}$ frequency range), by Deep Levels Transient Spectroscopy with electrical (DLTS) and optical (ODLTS) injection (Polyakov et al., 2011) (for these measurements a completely automated setup based on HP4280A C-V/C-t meter with external pulse generator HP8112A and the oxford instruments gas-flow cryostat was employed; at each point the entire capacitance relaxation curve was stored and the transients could be processed with any chosen set of time windows providing all the necessary information from one temperature scan), by Micro-Cathodo Luminescence (MCL) spectra measurements and by MCL imaging, by Electron Beam Induced Current (EBIC) imaging and by low temperature $\mathrm{C}-\mathrm{V}$ measurements performed in the dark and after illumination (those were done to determine the concentration of deep electron traps with a barrier for capture of electrons and to estimate the density of deep hole traps located below the fermi level (Lee et al., 2011; 2012; Polyakov et al., 2011). Schottky diodes for these experiments were prepared by vacuum evaporation of $\mathrm{Ni}$ through a shadow mask (diode diameter typically $0.8 \mathrm{~mm}$ ). Steady-state and pulsed illumination in $\mathrm{C}-\mathrm{V}$ measurements and ODLTS was provided by high-power Light Emitting Diodes (LEDs) with the peak wavelength ranging from 660 $\mathrm{nm}$ to $365 \mathrm{~nm}$. Experimental setups were described previously (Lee et al., 2012; Polyakov et al., 2011).

\section{RESULTS}

The electrical and optical properties of group 1 samples with a higher growth temperature were radically different from the properties of group 2 samples. First of all, the concentration of residual donors in group 1 samples was more than an order of magnitude higher than for group 2 samples: About $10^{17} \mathrm{~cm}^{-3}$ versus $5 \times 10^{14}-10^{16} \mathrm{~cm}^{-3}$. This is illustrated by Fig. 1 comparing the $1 / \mathrm{C}^{2}$ versus $\mathrm{V}$ characteristics for two typical samples ( $\mathrm{C}$ here stands for capacitance, $\mathrm{V}$ is the applied bias). In group 1 the plot was linear, with the voltage intercept of $0.9 \mathrm{~V}$ close to the Schottky barrier height of Ni. For group 2 samples, not only was the measured concentration lower when calculated from the slope of the linear portion of the plot, but the voltage intercept was very high, over $100 \mathrm{~V}$ in all studied samples, which indicates the presence of a thick highly compensated layer at the top this region is fully depleted of carriers even at $0 \mathrm{~V}$. Concentration profiles calculated from $\mathrm{C}-\mathrm{V}$ measurements give the thickness of this highly compensated totally depleted layer as 2-4.5 $\mu \mathrm{m}$. The donor concentrations quoted above refer to the deeper lying regions of group 2 samples closer to the substrate. The observed spread in concentrations in this region reflects the difference in compensation for different samples. We also observed a certain difference in the thickness of the fully depleted at $0 \mathrm{~V}$ highly compensated 
top portion of the films and noticed that the thickness of this portion showed some correlation with the residual donor concentration in the portion of the layer closer to the substrate: The thickness of the depleted layer was higher for samples with a higher density of residual donors in the near-substrate portion. At the moment we do not have a viable explanation of the observed behavior. Clearly, more experiments are needed here.

Deep traps spectra measurements for group 1, group 2 samples were quite straightforward. Fig. 2 and 3 present the DLTS spectra obtained for samples of two groups. With standard time windows settings commonly used in our DLTS experiments three electron traps with apparent activation energies $0.25 \mathrm{eV}$ (ET1), $0.35 \mathrm{eV}$ (ET2) and $0.65 \mathrm{eV}$ (ET3) could be detected (see the solid line spectrum in Fig. 2 measured for one of the group 1 samples with time windows settings of 100/1000 ms). With much longer time windows an additional trap with the apparent activation energy of $1 \mathrm{eV}$ (ET4) was observed (see the dashed line spectrum in Fig. 2 corresponding to the time windows of $1500 / 15000$ $\mathrm{ms}$ ). In all samples the ET1-ET4 electron traps concentrations were in the mid- $10^{13}-10^{14} \mathrm{~cm}^{-3}$ range. The traps presented in Fig. 2 and 3 are quite common for undoped n-GaN films grown by HVPE, MOCVD or MBE (Lee et al., 2012; Polyakov et al., 2011; Palmer, 2011; Pearton et al., 2013).

Deep hole traps spectra obtained for group 1 and 2 samples with intrinsic optical excitation (high-power 365-nm LED source) are presented in Fig. 4 and 5, respectively. For group 1, two hole traps with apparent activation energies $0.6 \mathrm{eV}$ and $0.9 \mathrm{eV}$ could be seen in the spectra measured with our standard time windows settings (Fig. 4, solid curve obtained for the time windows settings of 100/1000 ms). These spectra also pointed to the presence of some deeper lying hole traps whose peak could be reliably observed in the spectra measured with longer time windows (Fig. 4, dashed curve corresponding to the time windows of $2500 \mathrm{~ms} / 25000 \mathrm{~ms}$ ). The apparent activation energy of these hole traps was $1.2 \mathrm{eV}$. The hole traps in question have been reported previously for HVPE and MOCVD undoped n-GaN samples (Lee et al., 2012; Polyakov et al., 2011; Pearton et al., 2013). The $0.6 \mathrm{eV}$ hole traps parameters are close to the parameters of the H3 hole traps in HVPE GaN (Lee et al., 2012), for the $0.9 \mathrm{eV}$ trap the parameters are similar to the $\mathrm{H} 1$ hole traps in MOCVD and HVPE GaN (Polyakov et al., 2011). The $1.2 \mathrm{eV}$ hole traps resemble the H5 hole traps in HVPE
GaN (Lee et al., 2012). For all group 1 samples the concentrations of $\mathrm{H} 3, \mathrm{H} 1, \mathrm{H} 5$ hole traps were around $5 \times 10^{14} \mathrm{~cm}^{-3}$, with the aggregate density of hole traps measured in ODLTS close to $1.5 \times 10^{15} \mathrm{~cm}^{-3}$ for the sample whose ODLTS spectra are depicted in Fig. 4. As discussed in our previous papers the aggregate density of deep hole traps in $\mathrm{n}-\mathrm{GaN}$ can be also estimated from the persistent decrease of the voltage intercept in $\mathrm{C}-\mathrm{V}$ characteristics after illumination at low temperature (Lee et al., 2012; Polyakov et al., 2011). For group 1 samples the density of deep hole traps determined that way was $(2-2.5) \times 10^{15}$ $\mathrm{cm}^{-3}\left(2.3 \times 10^{15} \mathrm{~cm}^{-3}\right.$ for the sample in Fig. 4), in reasonable agreement with the ODLTS data.

For group 2 samples with a highly compensated layer at the top, DLTS and ODLTS spectra are shown in Fig. 3 to 5. In DLTS, the spread in the type and magnitude of the electron traps peaks between the different samples was quite significant. For some samples two major electron traps with activation energy $0.5 \mathrm{eV}$ (a new trap compared to group 1 samples, trap ET5) and $0.65 \mathrm{eV}$ (ET3 traps) could be detected with our standard windows settings (solid line in Fig. 3 corresponding to the time windows of 100/1000 ms for one of the group 2 samples, sample 2-1). For longer time windows a peak belonging to deeper electron traps with activation energy $1 \mathrm{eV}$ (ET4 traps, the dashed line in Fig. 3) could be detected. At the same time, for other group 2 samples, the signal from electron traps was markedly absent for the whole studied temperature range and the entire range of time windows settings. The determination of the traps density in group 2 films was more complicated because of the very non uniform distribution of the uncompensated shallow donors' density along the samples thickness. For measurements with applied reverse bias and the injection pulse amplitude not exceeding $0 \mathrm{~V}$, however, the donor density could be considered as relatively constant and could be taken from the slope of the linear portion of the $1 / \mathrm{C}^{2}$ versus $\mathrm{V}$ plot. In that case, an estimate of the traps density from the magnitude of the DLTS peaks could be done in the usual fashion when such peaks were detected. For sample 2-1 with the donor density calculated to be $10^{16} \mathrm{~cm}^{-3}$ from $\mathrm{C}-\mathrm{V}$ profiling, the traps densities in Fig. 3 were estimated as $7.3 \times 10^{13} \mathrm{~cm}^{-3}(0.5$ eV ET5 traps), $6.6 \times 10^{13} \mathrm{~cm}^{-3}(0.65 \mathrm{eV}$ ET3 traps $)$ and $7.3 \times 10^{13} \mathrm{~cm}^{-3}(1 \mathrm{eV}$ ET4 traps). These values are not radically different from the electron trap densities in group 1 samples. However, in other group 2 samples 
with much lower residual donor concentration no electron traps in detectable concentration were observed.

The situation with hole traps in group 2 samples was even more complicated. In that case optical injection occurs across the region with a highly non-uniform distribution of shallow donors. Hence, the hole traps signal can depend on both the penetration depth of the excitation light and the efficiency of ionization of deep hole traps with the light pulse of the given wavelength. For sample 2-1 discussed above, the spectra for intrinsic strong-absorption excitation with the highpower 365-nm wavelength LED and with the extrinsic weakly absorbed 400-nm wavelength LED are compared in Fig. 5. The spectra measured with 365nm LED excitation clearly demonstrate the presence of hole traps with activation energy $0.53 \mathrm{eV}$, similar to the $\mathrm{H} 2$ hole traps (Lee et al., 2012), hole traps with activation energy $0.6 \mathrm{eV}$ (H3), hole traps with activation energy $0.9 \mathrm{eV}(\mathrm{H} 1)$ and hole traps with activation energy $1.2 \mathrm{eV}$ resembling the H5 hole traps of Fig. 4, but showing a higher hole capture cross section. With extrinsic excitation the peaks of all low energy hole traps were strongly suppressed while in the high temperature range a new hole trap H6 with activation energy $0.94 \mathrm{eV}$ emerged. The reason for such a strong influence of the excitation light wavelength on the measured spectra has yet to be understood, but it seems reasonable to ascribe the effect to the difference in the deep hole traps types at different depths from the surface. For strongly absorbed 365-nm wavelength excitation probing the near-surface portion of the film, the $\mathrm{H} 2, \mathrm{H} 3$, H1 and H5 traps dominate, whereas for excitation with weakly absorbed 400-nm LED probing the deeper portion of the film the H6 hole traps are dominant.

Room temperature MCL spectra for several group 1 and group 2 samples are depicted in Fig. 6. One can clearly see the intense band edge luminescence line near $3.35 \mathrm{eV}$, the rather weak defect MCL band peaked near $2.5 \mathrm{eV}$ (the green band marked as "GB" in the figure), similarly weak defect $2.3 \mathrm{eV}$ yellow MCL band (marked "YB" in the figure) and a shoulder or true peak belonging to the defect MCL band near $1.9 \mathrm{eV}$ (marked as red band "RB" in the figure). Bands in Fig. 6 are well documented for undoped n-GaN films (Reshchikov and Morkoc, 2005). For the red band detailed studies on HVPE samples similar to the ones described in the present paper allowed to attribute the transition to acceptors located close to $E_{v}+1.13 \mathrm{eV}$ (Reshchikov et al., 2014). In earlier studies the green band and the yellow bands were ascribed to other deep acceptor centers with levels in the vicinity of $\mathrm{E}_{\mathrm{v}}+1 \mathrm{eV}$ based on the spectral dependence of the ODLTS peaks magnitude and on correlation of the peak magnitude in ODLTS and the relative peak intensity in MCL spectra (Lee et al., 2012; Polyakov et al., 2011; Pearton et al., 2013).

EBIC imaging of the sample surface revealed in all of the samples the presence of dark spot defects commonly associated with threading dislocations in GaN (Yakimov et al., 2007). The density of these defects was close to $10^{8} \mathrm{~cm}^{-2}$ for both group 1 and group 2 samples. In addition, we also observed in EBIC larger dark defects with characteristic density of $10^{7} \mathrm{~cm}^{-2}$ (Fig. 7 for one of the group 1 samples). Similar dark spots defects could be detected in band edge MCL images of the samples. The dark spots/dislocation density indicates a reasonably high crystalline quality of the studied samples given that they are relatively thin in so far as the HVPE films go (dislocation densities below $10^{6} \mathrm{~cm}^{-2}$ or even $10^{4} \mathrm{~cm}^{-2}$ can be obtained for layers with thicknesses exceeding $\sim 100 \mu \mathrm{m}$ (Yoshida et al., 2008; Sato et al., 2013; Fujito et al., 2009).

The diffusion length of non equilibrium charge carriers can be estimated from linear EBIC scans along the surface of the sample adjacent to the Schottky diode edge (Yakimov et al., 2008). Figure 8 presents such an EBIC linear scan for one of the group 1 samples (sample $1-1$, the same sample for which DLTS, ODLTS, MCL spectra are shown in Fig. 2, 4 and 6 and the EBIC image is presented in Fig. 7). Fitting of the observed EBIC signal intensity as a function of the distance to the Schottky diode edge gives the diffusion length for this particular sample as $1 \mu \mathrm{m}$. For group 2 samples the diffusion length estimated that way was $1.5 \mu \mathrm{m}$ (Fig. 8).

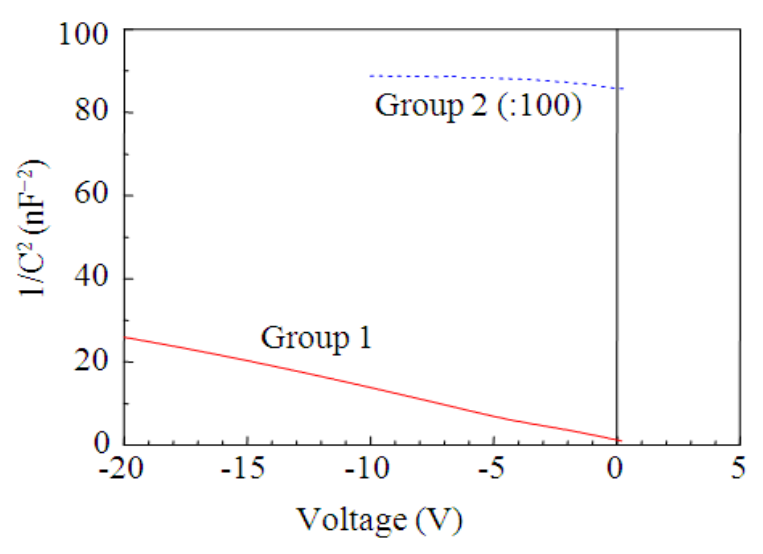

Fig. 1. Room temperature $1 / \mathrm{C} 2$ versus $\mathrm{V}$ plots for one of the group 1 samples (solid line, red) and group 2 samples (dashed line, blue). All figures are original figures not used elsewhere 


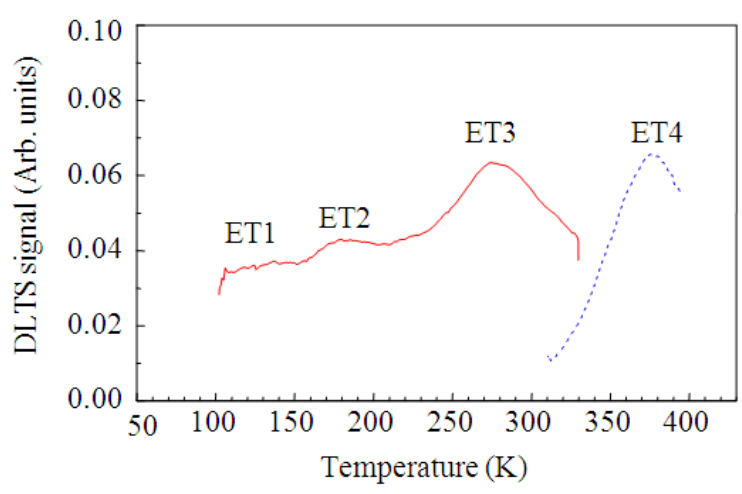

Fig. 2. DLTS spectra measured for one of the group 1 samples with reverse bias $-0.5 \mathrm{~V}$, forward bias pulse of $1 \mathrm{~V}$ and time windows $100 / 1000 \mathrm{~ms}$ (solid line, red) and 1500/15000 ms (dashed line, blue)

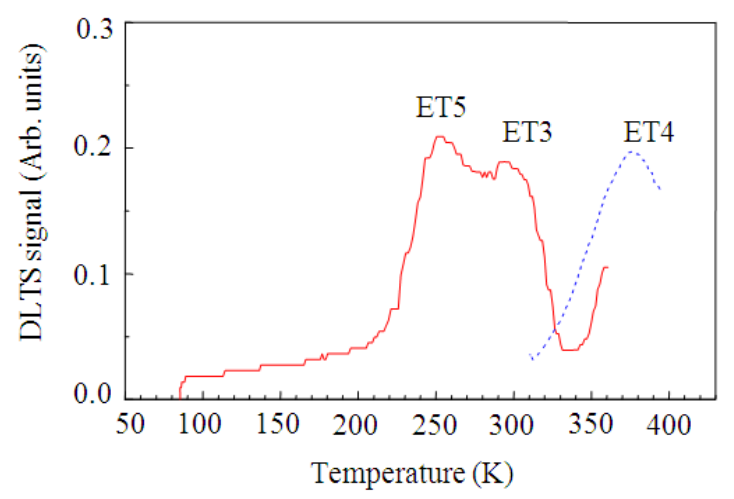

Fig. 3. DLTS spectra measured for one of the group 2 samples at $-1 \mathrm{~V}$, with the forward bias pulsed to $0 \mathrm{~V}$, with the time windows 100/1000 $\mathrm{ms}$ (solid line, red) and $1500 / 15000 \mathrm{~ms}$ (dashed line, blue)

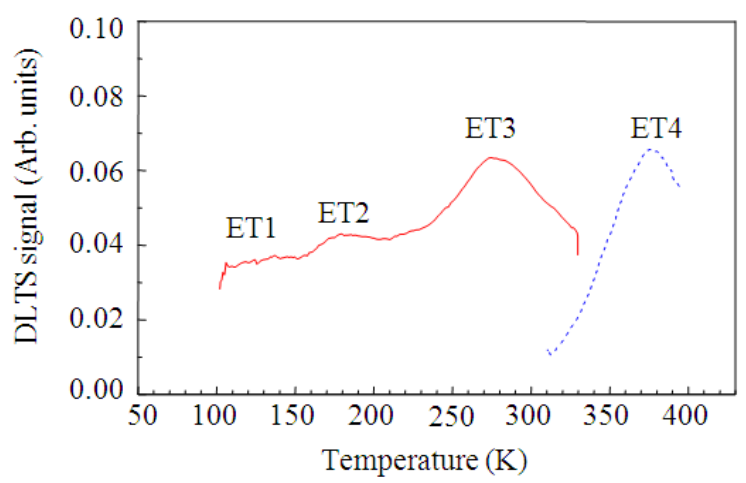

Fig. 4. ODLTS spectra measured for one of the group 1 samples at $-0.5 \mathrm{~V}, 365-\mathrm{nm}$ wavelength LED excitation and time windows $100 / 1000 \mathrm{~ms}$ (solid line, red) and 2500/25000 ms (dashed line, blue)

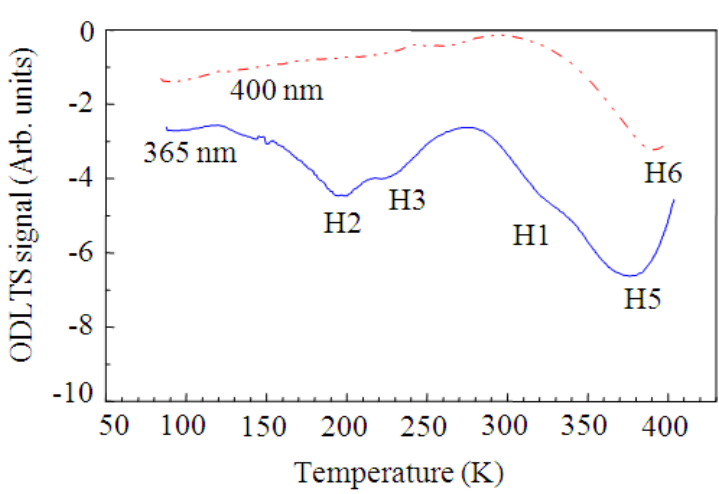

Fig. 5. ODLTS spectra measured for one of the group 2 samples at $-0.5 \mathrm{~V}$, with time windows $500 / 5000 \mathrm{~ms}$, with $365-\mathrm{nm}$ wavelength LED excitation (solid line, blue) and 400-nm wavelength LED excitation (dashed line, red)
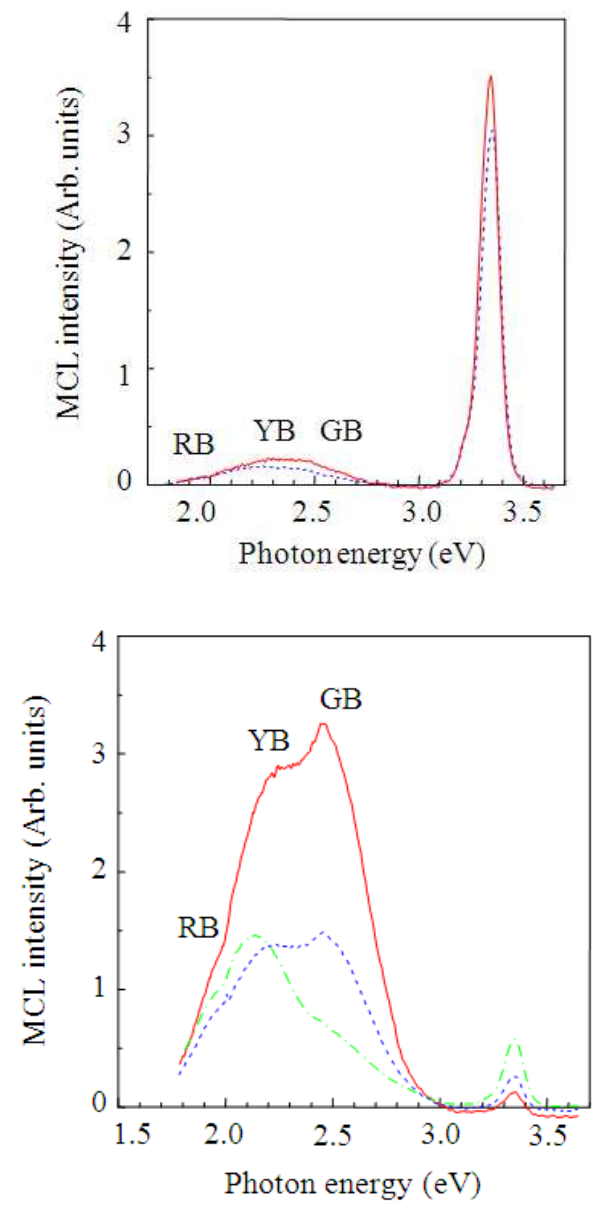

Fig. 6. Room temperature MCL spectra of two group 1 (on the left) and group 2 (on the right) samples 


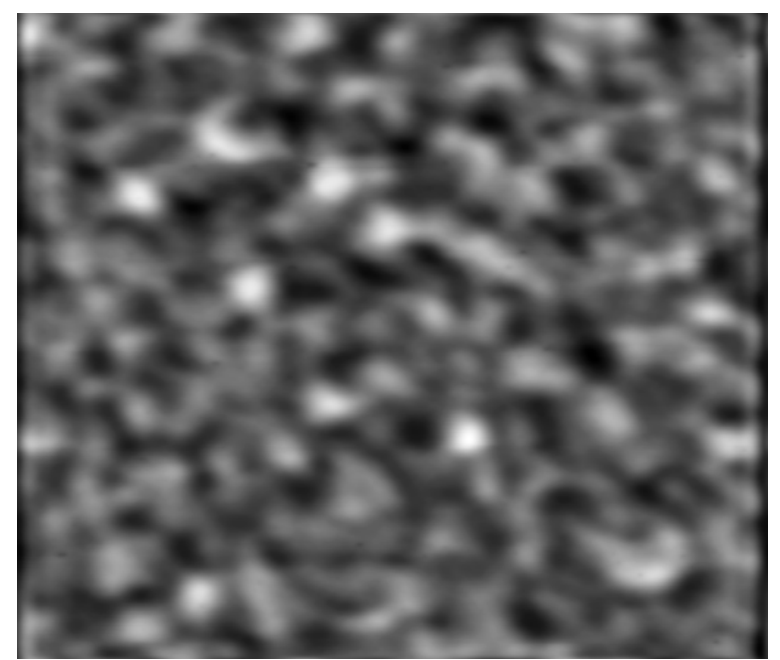

Fig. 7. EBIC image of one of the group 1 sample surface, the full figure size is $33 \times 33 \mu \mathrm{m}$.

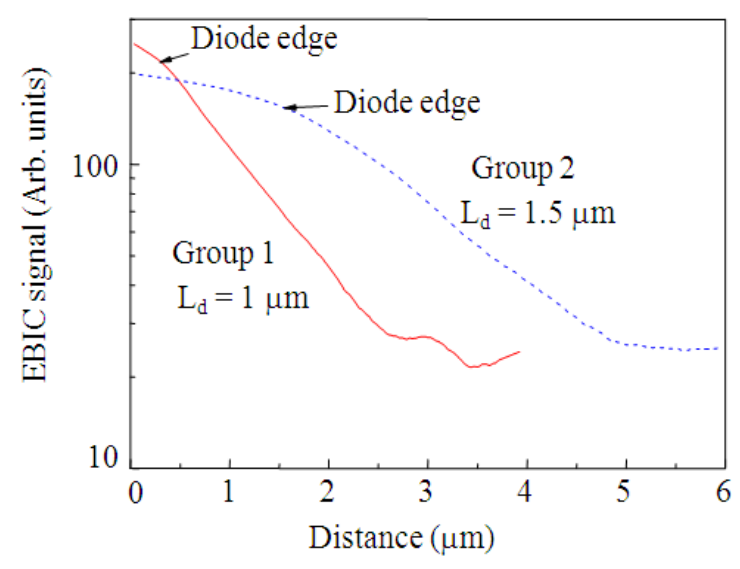

Fig. 8. EBIC line scans near the edge of the Schottky diode for one of the group 1 samples (solid line, red) and one of the group 2 samples (dashed line, blue)

It should be noted that the diffusion length values can also be determined from EBIC measurements by using the dependence of the EBIC collection efficiency on accelerating voltage of the probing electron beam (Yakimov et al., 2008). The values obtained that way were close to 0.1 and $0.15 \mu \mathrm{m}$ for group 1 and group 2 samples, respectively

\section{DISCUSSION}

Comparison of deep traps spectra in group 1 and group 2 samples shows that (a) the density of deep electron traps was on average lower in group 2 samples; (b) the hole traps detected in group 2 samples differed considerably for excitation with the strongly absorbed intrinsic light and weakly absorbed extrinsic light suggesting the non-uniform distribution of deep hole traps along the growth axis, with H6 hole traps dominating in the deeper parts of the films. At that the higher diffusion length measured by the two used methods for group 2 samples suggests the lower overall density of non-radiative recombination centers in group 2 samples. Radiative transitions detected in room temperature MCL spectra point in both groups of samples to the distinguished role of defect bands associated with deep acceptors with levels in the vicinity of $\mathrm{E}_{\mathrm{v}}+1 \mathrm{eV}$. However, for group 2 samples the intensity was re-pumped from the bandedge line into the defect green, yellow and red bands (Reshchikov and Morkoc, 2005; Reshchikov et al., 2014). This re-pumping is due to the much lower shallow donors and free electron concentrations causing a more prominent impact of recombination via the deep traps. At that, the integrated luminescence intensity was similar in both groups of samples indicating that one deals here with the bands intensity re-distribution and not with the enhanced impact of non-radiative recombination due to the deep traps.

In $\mathrm{GaN}$ it is well established that dislocations provide an important channel of non-radiative recombination (Dmitriev and Usikov, 2006; Pearton et al., 2013). It has been noted that the diffusion lengths in GaN films with various dislocation densities often times are determined by the average distance between the dislocations (or) mean dimensions of the dislocation cells for high dislocation densities when the dislocations form cellular structure (Chernyak et al., 2000). This correlation exists owing to the fact that dislocations are the main recombination agents in GaN. This does not seem to be the case for the samples studied here. Indeed, the dislocation densities, as measured by the dark spot defects were virtually the same for group 1 and group 2 samples. Yet, the measured diffusion lengths were markedly different suggesting a strong impact of recombination via deep defects associated with point defects andthe lower density of the latter in group 2 samples.

The origin of the strong difference in the diffusion lengths values determined from linear scanning and from the probing beam energy dependence in EBIC needs further study. The three obvious reasons to be considered are (a) the secondary luminescence leading 
to the appearance of long tails in EBIC decay curves with the distance from the Schottky diode edge; (b) the strong band bending at the surface of the samples caused by the fermi level pinning even outside the Schottky barrier and to electrons and holes spatial separation; and (c) the non-uniform distribution of point defects along the growth axis.

Finally, a few words need to be added regarding the possible origin of the larger dark spots in EBIC images as depicted by Fig. 7. The reason for their appearance seems to be related to the local difference in doping rather than to the local difference in the lifetime. At the moment we somewhat tentatively attribute these local variations to the donors segregation on screw dislocations. The ratio of screw and edge dislocations in our samples determined from selective etching and from x-ray data is quite close to the ratio of small and large dark spot defects.

\section{CONCLUSION}

We have shown that in the HVPE growth process reduced growth temperature at $850^{\circ} \mathrm{C}$ or so resulted in lower concentration of shallow donors and the formation of high-resistivity layer in the top portion of the films. The lower growth temperature also facilitates a decrease in the density of deep traps and an increase in the charge carriers diffusion lengths and lifetimes. The deep traps spectra are dominated by the electron traps with activation energies $0.25 \mathrm{eV}$ (ET1), $0.35 \mathrm{eV}$ (ET2), 0.5 $\mathrm{eV}$ (ET5), $0.65 \mathrm{eV}$ (ET3), $1 \mathrm{eV}$ (ET4) and by the hole traps with activation energy $0.53 \mathrm{eV}(\mathrm{H} 2), 0.6 \mathrm{eV}(\mathrm{H} 3)$, $0.9 \mathrm{eV}$ (H1), $0.95 \mathrm{eV}$ (H6) and $1.2 \mathrm{eV}$ (H5). The hole traps distribution along the growth direction seems to be non-uniform, with H6 hole traps dominating in the deeper parts of the films grown at higher temperature. Since previous experiments have been able to tie rather convincingly the $\mathrm{H} 1$ traps to gallium vacancy $\mathrm{V}_{\mathrm{Ga}}$ complexes with $\mathrm{Si}$ donors, $\mathrm{V}_{\mathrm{Ga}}-\mathrm{Si}$ and the $\mathrm{H} 5$ traps with $\mathrm{V}_{\mathrm{Ga}}$ complexes with oxygen donors, $\mathrm{V}_{\mathrm{Ga}} \mathrm{O}$ (Lee et al., 2011; 2012; Polyakov et al., 2011), one can speculate that the reason for both the decreased density of shallow donors and the decreased density of deep hole traps, particularly, $\mathrm{H} 1$ traps, is related to the lower concentration of $\mathrm{V}_{\mathrm{Ga}}$ formed at the lower growth temperature.

However too low growth temperature lower than $850^{\circ} \mathrm{C}$ may deteriorate crystalline and electrical quality of the grown layer presumably because of increasing point defects and increases background carrier concentration and deep traps. It looks there is an optimal reduced growth temperature in a combination with other growth conditions resulting in lower concentration of shallow donors however still keeping high crystalline quality of the layer.

In future work further reduction of the residual donor density to the level well below $10^{15} \mathrm{~cm}^{-3}$ and further decrease of the hole traps density to below $10^{14} \mathrm{~cm}^{-3}$ will be aimed by optimization of the growth conditions (in-situ substrate conditioning, growth rate modulation, growth temperature changing, V/III ratio and some others) and by improving the purification of the carrier gases and precursors used in the process. This should bring the grown undoped HVPE films quality to the level required by current day power rectifiers and by radiation detectors.

\section{ACKNOWLEDGMENT}

The work at NUST MISiS was supported in part by the Ministry of Education and Science of the Russian Federation in the framework of increase competitiveness program of NUST «MISiS» (№ K2-2014-055). The work at Chonbuk National University was supported by the Brain Pool program of Korean Government.

\section{REFERENCES}

Chernyak, L., A. Osinsky, G. Nootz, A. Schulte and R.J. Molnar et al., 2000. Electron beam and optical depth profiling of quasibulk GaN. Applied Phys. Lett., 77: 2695-2697. DOI: 10.1063/1.1319530

Dmitriev, V. and A. Usikov, 2006. Hydride Vapor Phase Epitaxy of Group III Nitride Materials. In: III-Nitride Semiconductor Materials, Feng, Z.C. (Ed.)., Imperial College Press, London, ISBN-10: 86094-636-4, pp: 1-40.

Fujito, K., S. Kubo, H. Nagaoka, T. Mochizuki and S. Nagao et al., 2009. Bulk GaN crystals grown by HVPE. J. Crystal Growth, 311: 3011-3014. DOI: 10.1016/j.jcrysgro.2009.01.046

Kurin, S., A. Antipov, I. Barash, A. Roenkov and Y. Makarov et al., 2013. CHVPE growth of AlGaNbased UV LEDs. Phys. Status Solidi, 10: 289-293. DOI: $10.1002 /$ pssc. 201200640

Lee, I.H., A.Y. Polyakov, N.B. Smirnov, A.V. Govorkov and S.J. Pearton et al., 2011. Deep electron and hole traps in neutron transmutation doped n-GaN. J. Vacuum Sci. Technol., 29: 041201-041201. DOI: 10.1116/1.3596571 
Lee, I.H., A.Y. Polyakov, N.B. Smirnov, A.V. Govorkov and S.J. Pearton et al., 2012. Electrical properties and radiation detector performance of free-standing bulk n-GaN. J. Vacuum Sci. Technol., 30: 021205021205. DOI: 10.1116/1.3690644

Lipski, F., 2010. Semi-insulating GaN by fe-doping in hydride vapor phase epitaxy using a solid iron source. Institute of Optoelectronics, Ulm University, Germany.

Liu, N., J. Wu, W. Li, R. Luo, Y. Tong, G. Zhang, 2014. Highly uniform growth of 2-inch GaN wafers with a multi-wafer HVPE system. J. Crystal Growth, 388: 132-136. DOI: 10.1016/j.jcrysgro.2013.11.023

Palmer, D.W., 2011. Electronic Energy Levels in GroupIII Nitrides. In: Comprehensive Semiconductor Science and Technology, Bhattarcharya P., R. Fornari and H. Kamamura, (Eds.)., Elsevier, Amsterdam, ISBN-10: 978-0-444-53153-7, pp: 390-447.

Paskova, T., D.A. Hanser and K.R. Evans, 2010. GaN substrates for III-nitride devices. 2010. Proceedings of the IEEE, (PIE' 10), IEEE Xplore Press, pp: 1324-1338. DOI: 10.1109/JPROC.2009.2030699

Pearton, S.J., R. Deist, F. Ren, L. Liu and J. Kim et al., 2013. Review of radiation damage in GaN-based materials and devices. J. Vacuum Sci. Technol., 31: 050801-050801. DOI: 10.1116/1.4799504

Polyakov, A.Y., I.H. Lee, N.B. Smirnov, A.V. Govorkov and S.J. Pearton et al., 2011. Comparison of hole traps in $\mathrm{n}-\mathrm{GaN}$ grown by hydride vapor phase epitaxy, metal organic chemical vapor deposition and epitaxial lateral overgrowth. J. Applied Phys., 109: 123701-123701. DOI: 10.1063/1.3599894

Reed, M.L., E.D. Readinger, C.G. Moe, H. Shen and V.A. Dmitriev et al., 2009. Benefits of negative polarization charge in $\mathrm{n}$-InGaN on $\mathrm{p}-\mathrm{GaN}$ single heterostructure light emitting diode with $\mathrm{p}$-side down. Phys. Status Solidi, 6: 585-588. DOI: 10.1002/pssc.200880401

Reshchikov, M.A. and H. Morkoç, 2005. Luminescence properties of defects in GaN. J. Applied Phys., 97: 061301-061301. DOI: 10.1063/1.1868059

Reshchikov, M.A., A. Usikov, H. Helava and Y. Makarov, 2014. Fine structure of the red luminescence band in undoped GaN. Applied Phys. Lett., 104: 032103032103. DOI: 10.1063/1.4862790

Reshchikov, M.A., A.A. Kvasov, M.F. Bishop, T. McMullen and A.A. Dmitriev et al., 2011. Tunable and abrupt thermal quenching of photoluminescence in high-resistivity Zn-doped GaN. Phys. Rev., 84: 075212-075212. DOI: 10.1103/PhysRevB.84.075212
Richter, E., N. Stoica, U. Zeimer, C. Netzel and G. Tränkle et al., 2013. Si Doping of $\mathrm{GaN}$ in Hydride Vapor-Phase Epitaxy. J. Elect. Mater., 42: 820-825. DOI: $10.1007 / \mathrm{s} 11664-012-2373-2$

Sato, T., S. Okano, T. Goto, R. Yao and H. Goto et al., 2013. Nearly 4-inch-diameter free-standing $\mathrm{GaN}$ wafer fabricated by hydride vapor phase epitaxy with pit-inducing buffer layer. Jap. J. Applied Phys., 52: 1-3. DOI: 10.7567/JJAP.52.08JA08

Usikov, A., O. Kovalenkov, V. Soukhoveev, V. Ivantsov and A.V. Davydov et al., 2008. Electrical and optical properties of thick highly doped p-type GaN layers grown by HVPE. Phys. Status Solidi, 5: 18291831. DOI: $10.1002 /$ pssc. 200778685

Usikov, A., V. Soukhoveev, O. Kovalenkov, A. Syrkin and V. Ivantsov et al., 2013. Accumulation of background impurities in hydride vapor phase epitaxy grown GaN layers. Jap. J. Applied Phys., 52: 08JB22-08JB22. DOI: 10.7567/JJAP.52.08JB22

Wu, Y.H., C.H. Lee, C.M. Chu, Y.H. Yeh and W.I. Lee, 2013. A simple growth method to produce a-plane $\mathrm{GaN}$ thick films by hydride vapor phase epitaxy. Jap. J. Applied Phys., 52: 08JB08-08JB08. DOI: 10.7567/JJAP.52.08JB08

Wang, Y., H. Xu, S. Alur, Y. Sharma and K.R. Evans et al., 2011. Electrical characteristics of the vertical GaN rectifiers fabricated on bulk GaN wafer. Phys. Status Solidi, 8: 2430-2432. DOI: 10.1002/pssc.201001158

Yakimov, E.B., P.S. Vergeles, A.Y. Polyakov, N.B. Smirnov and S.J. Pearton et al., 2007. Spatial variations of doping and lifetime in epitaxial laterally overgrown GaN. Applied Phys. Lett., 90: 152114-152114. DOI: 10.1063/1.2722668

Yakimov, E.B., P.S. Vergeles, A.Y. Polyakov, N.B. Smirnov and A.V. Govorkov et al., 2008. Donor nonuniformity in undoped and $\mathrm{Si}$ doped n-GaN prepared by epitaxial lateral overgrowth. Applied Phys. Lett., 94: 042118-042118. DOI: 10.1063/1.2840190

Yoshida, T., Y. Oshima, T. Eri, K. Watanabe and T. Mishima et al., 2008. Preparation of 3 inch freestanding $\mathrm{GaN}$ substrates by hydride vapor phase epitaxy with void-assisted separation. Phys. Status Solidi, 205: 1053-1055. DOI: 10.1002/pssa.200778431 